\title{
Análisis de impacto de políticas públicas para el desarrollo local en comunidades costeras y rurales: El caso de la pesca en San Blas, Nayarit
} Impact analysis of public policies for local development in coastal and rural communities: the case of fishing in San Blas, Nayarit.

\author{
Deyrith Alondra Buhaya Lora \\ Universidad Autónoma de Nayarit \\ deyrith.buhayal@hotmail.com \\ Héctor R Ramírez Partida \\ Universidad Autónoma de Nayarit \\ hector_2093@hotmail.com
}

\section{Resumen}

Para un sin número de comunidades y espacios locales en México, las actividades económicas primarias conforman la base de su crecimiento y desarrollo tanto económico como social. Esto es particularmente cierto en localidades costeras a lo largo y ancho de México, y dentro de esta realidad se encuentra la pesca en el estado de Nayarit. A nivel socioeconómico, la pesca esencialmente se ha convertido para muchas familias de bajos recursos en una fuente de nutrición, empleo e ingresos, de esta forma, esto representa para las comunidades costeras un reto clave para el sustento de millones de familias, no solo en México y Nayarit sino también en muchos países con economías emergentes que han llevado agresivos procesos de reconversión económica derivado de los impactos en la economía mundial. Sin embargo, la importancia socioeconómica por lo general se monitorea a nivel macroeconómico - PIB- instrumento que no refleja los ingresos de la 
pesca a nivel comunidad o familia (pesca en pequeña escala). Por esto, el presente estudio busca caracterizar la actividad pesquera como estimulador del crecimiento y desarrollo económico y social en el municipio de San Blas, Nayarit. Para lograr esta caracterización, este trabajo analiza el impacto de la implementación de políticas públicas orientadas a estimular la actividad pesquera en Nayarit sobre los niveles de la actividad económica del municipio de San Blas. En la etapa en que se encuentra el presente estudio, la evidencia muestra que dada la importancia socioeconómica del sector, el impacto de las políticas públicas sobre la actividad es todavía tangencial y subestima la oportunidad que representa para el desarrollo de San Blas, Nayarit.

Palabras clave: impacto, desarrollo local, pesca

\section{Abstract}

For a number of communities and local areas in Mexico, the primary economic activities are the basis for growth and both economic and social development. This is particularly true in coastal localities throughout Mexico, and within this reality is fishing in the state of Nayarit. A socioeconomic status, essentially fishing has become for many low-income families a source of nutrition, employment and income, thus this represents for coastal communities a key challenge for the livelihoods of millions of families, not only in Nayarit and Mexico but also in many emerging economies that have led aggressive processes of economic restructuring derivative impacts on world economy.

Key words: impact, local development, fishing

Fecha recepción: Julio 2012

Fecha aceptación: noviembre 2012

\section{Introducción}

Las actividades económicas primarias conforman la base del crecimiento y desarrollo tanto económico como social de un sin número de comunidades y espacios locales en 
México. Particularmente, en localidades costeras la pesca es una actividad que representa una fuente importante de alimentos, empleo y beneficios económicos para quienes se relacionan a este sector. En los últimos años, la pesca mundial se ha convertido en un sector de la industria alimentaria con una evolución dinámica y los estados costeros han procurado aprovechar sus oportunidades en respuesta a la creciente demanda internacional de pescado y productos derivados.

La pesca representa un elemento del desarrollo que toma lugar en zonas costeras de México y del mundo. Este elemento del desarrollo es importante de evaluar porque en la esfera alimentaria provee proteína de calidad a cientos de millones de personas, y son precisamente estas zonas las que capturan cerca del 45 por ciento de los recursos pesqueros destinados para el consumo humano (Poggie y Pollnack, 1991). Por lo tanto, a nivel socioeconómico, la pesca se ha convertido para muchas familias de bajos recursos una fuente de nutrición, empleo e ingresos. Esto representa un reto clave para el sustento de millones de familias no solo en México y Nayarit, sino también en muchos países con economías emergentes que han llevado agresivos procesos de reconversión económica. Así, este trabajo busca caracterizar la pesca como estimulador del crecimiento y desarrollo económico en el municipio de San Blas, Nayarit.

A pesar de los beneficios económicos, sociales y nutricionales de la pesca, se tiene poca información de la contribución de la pesca a los medios de subsistencia y economías en países en desarrollo. En este contexto es fundamental resaltar que, en principio, los pescadores y sus familias en las comunidades se caracterizan por vivir en condiciones de pobreza. Pero al mismo tiempo, se reconoce que la pesca en comunidades costeras y rurales puede generar desarrollo económico a nivel local. Y es en este aspecto, que este trabajo busca evaluar el impacto de las políticas públicas para la promoción de las actividades pesqueras sobre el desarrollo económico local, y así hacer una contribución para expandir de manera consistente el análisis científico de la información que rodea a la actividad pesquera en México, y en particular, en el municipio de San Blas, Nayarit. 
Actualmente, aunque organizaciones, nacionales, internacionales y no gubernamentales reconocen que la pesca es un instrumento clave para el desarrollo de las localidades costeras, los impactos de las políticas del sector subestiman su potencial de desarrollo y carece de líneas concretas para el fomento y el proceso de particulares, grupos, organizaciones logren el desarrollo económico y social en el caso particular de San Blas, Nayarit.

Con este objetivo el presente trabajo de investigación se estructurará de la siguiente forma. En la primera parte, se caracteriza la importancia social y económica de la pesca como base de crecimiento y desarrollo en localidades costeras y rurales y su importancia para la seguridad alimentaria, así como la situación de la pesca a nivel mundial y las organizaciones internacionales ligadas al sector. Por otro lado, en la segunda parte se describen las experiencias a nivel internacional, se analizan los sectores competitivos y algunos casos exitosos en la implementación de políticas en el sector pesquero para fomentar el desarrollo económico por medio del sector.Después, en la tercera parte se describe la situación actual de la pesca en México, su contribución económica al producto interno bruto, así como una revisión de las zonas pesqueras como han sido estructuradas por la Secretaría de Agricultura, ganadería, desarrollo rural y pesca para determinar los elementos que toma en cuenta el gobierno federal para diseñar e implementar políticas públicas de desarrollo del sector.En la cuarta parte se presenta una evaluación de impacto del sector pesquero en la economía del estado de Nayarit. Esto, permite resaltar las característicasque guarda el sector como una de las principales actividades económicas del municipio de San Blas y relacionarse con las políticas implementadas a nivel nacional, estatal y municipal para el fomento de la actividad pesquera.Finalmente, lo anterior permite desarrollar una breve discusión de los resultados empíricos encontrados en la revisión de las políticas públicas y los ejes sobre los que se estructuran estas para fomentar y promover el desarrollo sustentable de la actividad pesquera. 


\section{Relevancia del Sector Pesca}

La importancia de la pesca se puede observar a nivel mundial, ya que el consumo per cápita de pescado comestible alcanzo un nuevo máximo histórico en 2008, proporcionando ingresos a los pescadores de subsistencia en pequeña escala y alimentos a miles de millones de consumidores. La FAO (2010) publicó en el documento llamado “El estado mundial de la pesca y la acuacultura 2010" que el sector pesquero es una fuente de ingresos y medios de subsistencia para cientos de millones de personas en todo el mundo. El empleo en el sector ha aumentado en las últimas tres décadas con un índice de crecimiento de $3.6 \%$ desde 1980 . Además la pesca contribuye a la seguridad alimentaria en los países en desarrollo, reducción de la pobreza, al desarrollo y medio de sustento. La pesca se considera una actividad muy importante para el desarrollo sostenible a nivel mundial.El Código de Conducta para la Pesca Responsable de FAO en el artículo 1.2, en su párrafo introductorio, "reconoce la importancia nutricional, económica, social, cultural y ambiental de la pesca". A su vez el artículo 6.2 estipula que la ordenación de la pesca incluye "la seguridad alimentaria, el alivio de la pobreza y el desarrollo sostenible".

La pesca es una de las actividades del sector primario, que conlleva otros procesos productivo-comerciales, como: industrialización o transformación, transporte, consumo humano y otras actividades que son de potencial beneficio a las comunidades aledañas, permitiendo ser un motor para el desarrollo de los tres sectores productivos, como son explotación-transformación, comercialización y prestación de servicios (Bravo, Martínez, Morales y Ramírez, 2009).Se estima que por cada persona empleada en la pesca existen otros tres puestos de trabajo en actividades secundarias, incluida la fase posterior a la captura, con un total de 180 millones de empleos en toda la industria pesquera. Además cada trabajador tiene a su cargo tres dependientes o familiares, por lo tanto el sector primario y secundario respaldan los medios de subsistencia de un total de 540 millones de personas, el 8.0 \% de la población mundial (FAO, 2010). El empleo en el sector pesquero ha aumentado más rápido que la población y que el empleo en la agricultura 
tradicional.Según la FAO, el 90 por ciento de los 38 millones de personas que se dedican a la pesca en todo el mundo pueden clasificarse como productores en pequeña escala. Se calcula que otros 100 millones de personas son empleadas directa o indirectamente en la pesca y acuicultura en pequeña escala. Además, hay millones de otros habitantes del medio rural que participan de forma estacional u ocasional.La mayoría de los pescadores y acuicultores viven en países en desarrollo, principalmente en Asia, continente que ha experimentado el mayor incremento en las últimas décadas. En 2008 el $85.5 \%$ de los pescadores y acuicultores vivían en Asia, seguida de África (9.3\%), América Latina (2.9\%), Europa (1.4\%), América del Norte (0.7\%) y Oceanía (0.1\%). Siendo China el país con mayor número de pescadores y acuicultores.

\section{Experiencias Internacionales en el Sector Pesca}

La Organización de las Naciones Unidas para la Alimentación y la Agricultura (FAO) reconoce su importancia nutricional, económica, social, cultural y ambiental y los intereses de todos aquellos que se relacionan con el sector. Debe llevarse a cabo de manera responsable, ya que se reconoce su importancia nutricional, económica, social, cultural y ambiental. La pesca se ha convertido en un sector fundamental para las economías en desarrollo debido a que permite mejorar los medios de subsistencia, las exportaciones, la seguridad alimentaria y el crecimiento económico. El procesado y la distribución de alimentos del mar representan una fuente importante de empleo especialmente para los países en desarrollo.A nivel internacional los sectores competitivos incluyen: el aumento de Chile como un importante productor de salmón del Atlántico, el desarrollo de las jaulas en alta mar para la dorada en el Mediterráneo, la expansión del cultivo de bagre de canal en los EE.UU. y la creciente competencia de Vietnam, el aumento de Asia y América Latina en el suministro de tilapia a los mercados norteamericanos, y dentro de países, cambio gradual de la producción de una región a otra, como por ejemplo, en Tailandia (bagre y camarones) y los EE.UU. (bagre de canal), 
como las ventajas comparativas quedado claro.El crecimiento regional en la acuicultura, tanto dominado por China en las últimas décadas, de acuerdo con las cifras registradas, es probable que cambie con la oportunidad de cambiar, internacional la inversión y la ampliación de una base de conocimientos y de su movilidad. Habrá una tendencia creciente hacia las áreas con buenos recursos naturales, el potencial de producción de menor costo y / o el acceso a los crecientes mercados internacionales o regionales, y un fuerte énfasis en el crecimiento económico y el comercio (Muir et al. 1999b). La pesca ha evolucionado másallá de su contribución al crecimiento y empleo ya que es fuente importante de la seguridad alimentaria por la demanda de nutrientes a nivel mundial, por ejemplo, el $37 \%$ de la producción total de productos pesqueros se incorporan al mercado internacional para el consumo humano y animal. En este flujo de comercio global China junto con Indonesia desempeñan un papel protagónico en la industria pesquera. En 2006, para la FAO el $79 \%$ de la producción pesquera mundial provino de los países en desarrollo, los cuales han promovido la generación e implementación de política pública de impulso al sector, y así convertirse en proveedores del comercio mundial. Específicamente,estas políticas han estado encaminadas a fortalecer laadministración pesquera, suestructura funcional, y el ordenamiento pesquero. Por ejemplo, Islandia, Nueva Zelanda y Namibia son países que representan experiencias exitosas en el fortalecimiento de los sistemas de derechos pesqueros e incentivos para una administración responsable. En el Sudeste asiático se tiene la promoción de la "pesca basada en los derechos" sustentada en la Resolución sobre la pesca sostenible para la Seguridad Alimentaria en los países de la ASEAN (Asociación de naciones del Sudeste asiático, por sus siglas en ingles). En África en la cumbre sobre "Pescado para todos" (Nigeria, 2005), los jefes de Estado de la NEPAD (Nueva Alianza para el Desarrollo de África) se aprobó la promoción de la pesca basada en derechos. A su vez en Perú la pesca se está desplazando hacían el enfoque basado en derechos y se espera financiar una red de protección social para los pescadores.La Comunidad Europea el 8 de Noviembre de 2000 a través de la Comunicación al Consejo y Parlamento Europeo estipuló que, al sector pesquero le corresponde desempeñar un papel considerable en la lucha contra la pobreza, 
objetivo principal de desarrollo de la Comunidad Europea. En el contexto de la Comunidad Económica Europea, el diseño de políticas de promoción del sector pesca incluye diferentes niveles de gobernabilidad, por esto la comunidad ha buscado garantizar la coherencia entre las actividades relacionadas con la pesca para complementar las políticas de la Comunidad y la de los Estados miembros. Los principios del bloque económico europeo seresumen a continuación:

- Solidaridad con los países en desarrollo

- Interés comercial, manteniendo la importación de grandes cantidades de pescado en respuesta a la demanda de los consumidores europeos y garantizando la protección de los consumidores

- El interés económico y social, apoyando a determinadas comunidades de pescadores europeos mediante la continuación de actividades pesqueras en las aguas de terceros países

- La preocupación medioambiental, ya que los océanos y los mares son un recurso mundial y patrimonio común de la humanidad.

Estos objetivos fueron elaborados por la Comisión por una parte, para los países en desarrollo en los que la pesca es una prioridad en la estrategia de desarrollo y, por otra, para los países que han firmado un acuerdo pesquero con la Comunidad.

\section{Estado actual de la pesca en México}

La pesca en México tiene un valor económico, social y alimentario con fuertes impactos regionales en la que su potencial ha contribuido a la solución de problemas alimentarios y de generación de empleos. Su gran potencial radica en que México cuenta con 11, 592 kilómetros de litorales de los cuales 8475 corresponden al Litoral del Pacifico y 3117 al 
Golfo de México y Mar Caribe (INEGI, 2010), incluyendo islas y una zona económica exclusiva (ZEE) de 3 millones de kilómetros cuadrados. La Secretaria de Agricultura, Ganadería, Desarrollo Rural, Pesca y Alimentación (SAGARPA) estimó que el sector pesquero y acuícola aportó en 2009, 1.7 millones de toneladas de productos marinos, con un incremento del 10 por ciento en relación a 2008 y situó a México como décimo séptimo productor en el mundo. En 2010, según datos de la SAGARPA, el sector pesquero produjo $0.87 \%$ del PIB nacional y empleó el $0.65 \%$ de la población ocupada nacional (equivalente a 276,185 personas). Es decir, 273,187 personas se emplearon en actividades de pesca, 250,159 de ellas en captura marítima y 23,028 personas en sistemas controlados ó acuicultura. Debido a la naturaleza de la actividad y a la estructura sociodemográfica de nuestro país -que cuenta con numerosas comunidades rurales- este sector registra una fuerte actividad informal tanto en litorales como en aguas continentales (Juárez, et al. 2007). De acuerdo con autores como Juárez, Escobar y Luna (2007) la pesca en México se realiza en tres áreas geográficas principales: 1) litoral del Pacífico; 2) litoral del Golfo y El Caribe; y 3) aguas continentales.Cifras estimadas de 2006 (CONAPESCA) muestran que en el litoral del Pacífico se realizó la captura del 79\% del volumen de producción pesquera; en el litoral del Golfo y el Caribe se concentró alrededor del $19 \%$ del volumen y sólo $2 \%$ en aguas continentales.

La actividad pesquera, por su naturaleza, concentra gran parte de la actividad en algunos estados del país (Sonora, Sinaloa, Baja California Sur, Nayarit, Veracruz) -dentro de los cuales se ubica la zona de estudio- con importantes impactos regionales. En los últimos años, algunas especies de elevado valor en el mercado como el camarón, el langostino, la jaiba, el abulón y la tilapia han mostrado una mayor demanda en los mercados internacionales representado excelentes oportunidades de mercado, y otros más tradicionales (como el atún y la sardina) continúan enfrentando una fuerte demanda en el mercado interno y en menor medida en el mercado internacional.La Comisión Nacional de Acuacultura y Pesca (CONAPESCA) por su parte, define para la República Mexicana cinco 
regiones pesqueras, y acuícolas acorde con su vocación, sus características y sus oportunidades de desarrollo (SAGARPA, 2007):

Región I: Pacífico Norte (Baja California hasta Nayarit)

Región II: Pacífico Centro Sur (Jalisco hasta Chiapas)

Región III: Norte del Golfo de México (Tamaulipas y Veracruz)

Región IV: Golfo de México y Caribe (Tabasco hasta Quintana Roo)

Región V: Centro

El fortalecimiento de esta regionalización administrativa fue notoria entre los años 1970 y 2010, cuando a nivel agregado, México desarrolló el sector pesquero a diferentes niveles, por ejemplo, se logró la ampliación de la infraestructura pesquera, el desarrollo de nuevas pesquerías y el incremento del consumoper cápita $(12.81 \mathrm{~kg})$ de productos pesqueros para el 2010. En 1976 se estableció la ZEE y se ratificó en 1982 en la CONVEMAR. En 1995 se acuerda el Código de Conducta para la Pesca Responsable de la FAO. Derivado de esto México implementa instrumentos de política pesquera a partir de 1995 y desarrolla normas oficiales.La Comisión Nacional de Acuacultura y pesca en su informe "La pesca y la acuacultura en México: papel, logros y principales retos" menciona que México es un país con gran potencial para de la acuacultura y la maricultura que podrá desarrollar en los próximos años, actividades que son una oportunidad para el crecimiento y el desarrollo económico de la nación. Actualmente, ocupa el lugar 16 en producción pesquera a nivel mundial y la posición 26 en acuacultura (Anuario estadístico de pesca y acuacultura, 2010).De acuerdo con proyecciones de SAGARPA, para el año 2030 laproducción estará alrededor de 2.75 millones de toneladas anuales, este escenario se sostiene bajo el supuesto de contar con un sector ordenado que contribuya a duplicar la producción nacional de alimentos.

Aunado a esto, la CONAPO estima que en 2030 habrá en México 121 millones de personas y si la producción pesquera y acuícola no crece -no se cumple el escenario mencionadoes muy probable que la oferta de mariscos sea insuficiente.Por lo tanto, de acuerdo a la 
CONAPESCA como órgano desconcentrado de la SAGARPA, tiene la función de implementar políticas, programas y normatividad que conduzcan el desarrollo sustentable del sector pesquero y acuícola del país y organizando su misión bajo los objetivos estratégicos de: contribuir a mejorar las condiciones socioeconómicas de las comunidades rurales y pesqueras marginadas, incrementar la contribución de los productos pesqueros sanos, incentivar el desarrollo, aprovechar de forma ordenada y sustentable los recursos y fortalecer la operación y estructura institucional del sector pesquero.

Para sustentar la actividad la CONAPESCA define tres ejes rectores:

- Ordenamiento. Corregir los problemas estructurales de sobre capitalización, sobreocupación y sobre explotación.

- Crecimiento. Desarrollar recursos potenciales: pesca en aguas interiores y acuacultura.

- Competitividad. Fortalecer el sistema producto y la eco-eficiencia.

Todo esto, siguiendo la normatividad del Código de Conducta para la Pesca Responsable (FAO) y considerando las propuestas técnicas de FAO y CONAPESCA.

Conforme a la LGPAS las acciones de ordenamiento son un conjunto de instrumento de Política Pública:

- Permisos y concesiones

- Normas y regulaciones

- Programas de ordenamiento pesquero

Programas:

- Ordenamiento por recurso estratégico

- Ordenamiento ribereño

- Reducción al esfuerzo pesquero 
- Ordenamiento acuícola

- Vinculación productiva

Acciones de ordenamiento:

- Identificación y control de esfuerzo pequero

- Monitoreo de cuotas de captura en algunas pesquerías

- Implementación de métodos económicos y modernos de embarcaciones menores

- Utilizar innovaciones y métodos basado en la mejor ciencia disponible

\section{La pesca en Nayarit}

La costa de Nayarit se caracteriza por 1,600 kilómetros de litoral, presencia de esteros y lagunas, siendo la más grande la Laguna de Agua Brava. La producción pesquera total del estado ha tenido una gran mejor desde el año 2000 sin embargo a nivel región del Golfo de California, es estado ocupa una pequeña proporción de la producción pesquera de los cinco estados.

\section{Tabla 1. Características del municipio de San Blas, Nayarit.}

Región hidrológica Huicicila RH13, cuenca Huicicila-San Blas y subcuenca San Blas. 72.3\% de la superficie municipal. RH12 Lerma-Santiago, cuenca: rio Santiago-Aguamilpa

Se localiza en la zona ecológica del Trópico Seco, provincia del Delta del Rio Grande de Santiago y sistema eco geográfico de San Blas

Costa noroccidental del Pacifico Mexicano dentro de "Marismas Nacionales" (WWF) 10,000 ha (7.1\%) pertenece a San Blas

Ecosistema de Humedales. Sistema más productivo de la biosfera

Complejo de humedales Tecapán-Agua Brava-Marismas Nacionales, entre Sinaloa y Nayarit: canales de madera, llanuras de inundación, lagunas, manglares, esteros, lagunas costeras y 
marismas. Sistema más importante del Pacifico Mexicano

Manglar más extenso del Pacifico Mexicano

Clima cálido subhúmedo con lluvias en verano

Fuente: Elaboración propia a partir de datos publicados en INEGI, 2011.

San Blas es el municipio que cuenta con el mayor número de centros acuícolas de acuerdo con datos publicados por SAGARPA en el documento llamado Panorama Agrícola y Pesquero 2011.El municipio de San Blas representa el 3\% de la superficie estatal, es la costa noroccidental del pacifico mexicano clasificado dentro de "Marismas Nacionales" (WWF) y cuenta con el sistemas de humedales el cual es el sistema más productivo de biosfera, además cuenta con el manglar más extenso del pacifico mexicano. La población económicamente activa (PEA) representa el $33.8 \%(14,148$ habitantes) de la población, 12,320 son hombres, 1,828 son mujeres. $51.4-\%$ de la PEA se encuentra empleada en el sector primario, $36.0 \%$ en el terciario y el $11.4 \%$ en el sector secundario. El sector primario de acuerdo con el Sistema de Clasificación Industrial de América del Norte (SCIAN) comprende: Agricultura, Ganadería, Aprovechamiento; Pesca.

El estado ocupó en 2009 el cuarto lugar nacional por su volumen de producción de camarón, robalo y ostión y el quinto productor de mojarra. La pesca de camarón es la mayor relevancia del estado con un valor de 304 millones 889 mil pesos equivalentes a 8 mil 571 toneladas de las cuales el $97.2 \%$ se capturo en tres municipios: Tuxpan (41.8\%); Tecuala (28.5\%) y San Blas (26.9\%). Durante el 2000 y 2009 la producción de camarón tuvo un crecimiento anual promedio de 7.1\%, sin embargo en 2009 sufrió una contracción de 7.4\%. En cuanto a la pesca de mojarra se observa una tasa anual de $17.1 \%$ creciendo más allá del cien por ciento, Tepic contribuyo con el $67.9 \%$ del valor de la mojarra. La producción de robalo creció a una tasa anual de $11.6 \%$ de 2000 a 2009, con un crecimiento a $23.6 \%$ para el último año. San Blas, Santiago Ixcuintla, Tuxpan y Tecuala aportaron el total de la producción. El ostión fue la cuarta especie pesquera en 
importancia, Santiago Ixcuintla y San Blas aportaron el 91.2\% de la producción (SAGARPA, 2011).Nayarit fue el decimo primer estado en importancia pesquera por el volumen de producción en 2009 las especies más importantes fueron el camarón, la mojarra, el robalo y el ostión. Los municipios dedicados al sector son: San Blas, Tuxpan, Bahía de Banderas, Santiago Ixcuintla, Tecuala y Tepic (SAGARPA, 2011).

Tomando en cuenta lo anterior, el municipio de San Blas, Nayaritpudieraencontrarse entrelos líderes en producción pesquera no solo del Estado, sino también del Golfo de California, dadas las características mencionadas anteriormente, sin embargo, aunque se reportan avances en producción, los resultados no son los proyectados para una zona con características productivas lo que hace cuestionar ¿Qué papel desempeñan las políticas públicas enfocadas al sector?, si existen los organismos gubernamentales a nivel nacional cuyas normas y políticas están bien definidas y alineadas a los requerimientos técnicos de la FAO, ¿en qué parte se encuentra el fallo? ¿las deficiencias son a nivel gubernamental o particular o ambos?

Para responder las preguntas anteriores se llevó a cabo una revisión de las políticas enfocadas al sector pesquero implementadas a nivel municipal y su alineación con los planes estatales y nacionales.En el Plan Estatal de Desarrollo 2005 - 2011, se estableció el Programa Estatal deDesarrollo Rural y Encadenamiento Productivo Agropecuario, Acuícola-pesquero y Forestal-Silvícola (PREDEREP) con el objetivo de promover el desarrollo y la competitividad del sector y brindar bienestar a los nayaritas. El plan se elaboró a partir del reconocimiento de que el sector primario representa el punto base de partida para impulsar el desarrollo (Plan Estatal de Desarrollo Nayarit, 2005).El Plan Estatal de Desarrollo (PED) establece que la prioridad es el mejor aprovechamiento de los potenciales en cada una de las regiones de acorde a su vocación natural. Por su parte, el Plan Nacional de Desarrollo 2007 - 2012 (PND), define al sector agropecuario y pesquero como estratégico y prioritario para el desarrollo del país, como proveedor de alimentos consumidos por familias y por otro lado, como abastecedor de materias primas para las 
industrias manufactureras y de transformación, lo que lo posiciona como un importante generador de divisas al mantener un dinamismo exportador.El Plan Nacional de Desarrollo 2007-2012 (PND) está organizado en cinco ejes de política pública. Del eje 2, Economía competitiva y generadora de empleos, y de sus objetivos 7, 8, 9, 10 y 11 se desprende el Programa Sectorial de Desarrollo Agropecuario y Pesquero (PSDAP). Estos objetivos señalan, entre otros aspectos, la necesidad de asegurar alimento para toda la población mexicana, elevar el nivel de desarrollo humano y patrimonial de las comunidades de zonas rurales y costeras mediante la diversificación de las actividades económicas. Asimismo, garantizar la certeza jurídica y el impulso a la modernización integral del sector pesquero para hacerlo competitivo. El Programa Sectorial de Desarrollo Agropecuario y Pesquero (PSDAP) se organiza alrededor de cinco objetivos y estrategias para orientar el desarrollo del sector pesquero y acuícola. Estos objetivos son acordes con el PND que plantea la integración de las zonas rurales de alta y muy alta marginación a la dinámica del desarrollo nacional. Plantea el fortalecimiento de la acuacultura rural mediante proyectos de inversión de pequeña escala en aguas interiores y litorales, para crear unidades de producción acuícola que contribuyan a mejorar la alimentación de la población y fomentar la participación social en las actividades económicas.El actual PED 2011-2017 estructura sus políticas bajo tres ejes: gobernabilidad, calidad de vida y desarrollo integral. El sector pesquero corresponde al eje de desarrollo integral, cuyo objetivo consiste en sentar las bases para incrementar la competitividad del estado de Nayarit en el contexto Nacional e Internacional.Denomina las oportunidades estratégicas de desarrollo identificando siete clúster o cadenas de valor, ubicando al sector pesquero en el sexto lugar bajo el nombre de "productos del mar y acuacultura".La línea concreta de acción es el desarrollo de camaronicultura orgánica. Las prioridades del PED actual son el sector energético y turístico; dejando de lado a la pesca y su importancia en comunidades costeras y rurales, a pesar de estar definida como un sector prioritario por el Plan de Nacional de Desarrollo.Las estrategias para el crecimiento económico Sectorial y Regional se mencionan de forma general, sin definir acciones concretas para cada sector. En Organización de los sectores menciona lo siguiente: 
- Organización de productores

- Consolidar las figuras asociativas de productores

- Impulsar y fortalecer diversas formas de organización

- Apoyos institucionales:

- Eficientar el uso de los apoyos públicos a productores

- Apoyar la generación de proyectos productivos

- Reforma del marco jurídico: agropecuario, pesquero y forestal

El Congreso del Estado decreto el 25 de Mayo de 2011 la Ley de Pesca y Acuacultura Sustentables para el Estado de Nayarit donde estable los siguientes objetivos:

I. Establecer las bases para el fomento y desarrollo sostenible de la pesca y la acuacultura en el Estado;

II. Regular la planeación y el ordenamiento de las actividades pesqueras y acuícolas de la Entidad;

III. Fomentar y garantizar la sanidad e inocuidad pesquera y acuícola;

IV. Impulsar la organización y capacitación de los pescadores y acuicultores para ampliar sus posibilidades de acceso al crédito para la producción;

V. Promover y apoyar la investigación científica y tecnológica en la materia;

VI. Promover las acciones de coordinación entre autoridades estatales y municipales, en donde se garantice la participación de los pescadores y acuicultores y sus organizaciones, y

VII. Organizar y promover la comercialización de la pesca y la acuacultura, así como de sus bienes y servicios. 
Alineado a estos objetivos por su parte el plan de desarrollo municipal específicamente en el sector pesquero plantea que la economía del municipio se basa en las actividades agropecuarias, pesqueras y turísticas. San Blas cuenta con una gran variedad de especies de escamas con posibilidad de exportación comercial, presenta condiciones para producir y explotar camarón, ostión, almejas, jaibas, porque cuenta con un sistema estaurino alrededor de 3000 hectáreas. Las estrategias para lograr un mejor aprovechamiento de potencial productivo son reforzar la infraestructura y los sistemas de producción en las fases de captura y transformación de los productos pesqueros, al igual que los mecanismos de comercialización existentes, consolidar la organización del sector pesquero, a través, de asesorías, técnicas, capacitación y financiamiento. Mejorar los servicios de regulación y vigilancia. Consolidar el puerto de San Blas como un centro pesquero industrial.

\section{Discusión de resultados}

En México, la política federal para el sector pesquero y su desarrollo sustentable estáorganizada sobre ejes estratégicos incluyendo integración de cadenas y agregación de valor. No se puede negar que existen avances y una creciente de demanda de la industria a nivel internacional, nacional y estatal, pero al comparar los resultados de la producciónpesquera en el municipio de San Blas con lo determinado en las políticas y estrategias y líneas de acción, hace evidente una realidad muy distinta de los resultados con las políticas y escenarios esperados. Entonces podría decirse que existe un fallo en la implementación, ejecución y control de las políticasdestinadas a fomentar el desarrollo del sector pesquero. Las políticas hacen esperar éxitos futuros pero pareciera que no ofrece opciones reales a la industria pesquera, pues la mayoría de estas políticas fueron diseñadas en base a avances experimentales solamente.También, existen avances por resaltar respecto al trabajo de la SAGARPA y la CONAPESCA, en sus desarrolloshan identificado la integración de cadenas productivascomo una estrategia viable para aumentar la competitividad de las empresas individuales, ante los retos de los mercados globales. Un primer esfuerzo en este sentido, es el apoyo para laconformación de Comités 
Sistema Producto (CSP) por especie y entidad federativa.Hasta el momento hay CSP conformados para tilapia, bagre, ostión, camarón de cultivo ytrucha. De estos, se ha tenido mayor avance con los CSP de tilapia. En 2004 seconformaron los primeros comités estatales de tilapia en Jalisco, Tabasco y Yucatán,seguidos por los de Colima, Sonora, Veracruz, Sinaloa y Oaxaca, y están por hacerloNayarit y Campeche. Sin embargo todas estas organizaciones son de formación y participación voluntaria, por lo que es importante que el productor o pescador tenga claro cuáles son los beneficios que se pueden alcanzar para que se incentive su participación. El sector pesquero (marítimo y acuícola) en México tiene un elevado potencial, en particular la acuicultura se perfila como una actividad con buenas posibilidades tanto a nivel comercial en la colocación de productos de alto valor en el mercado internacional, como también a nivel artesanal en su papel de generadora de ingreso para comunidades en condiciones de pobreza. La gestión gubernamental ha emprendido acciones estratégicas para fortalecer el sector, entre las que se encuentran: la regulación de la actividad, de definición de lineamientos generales de una explotación sustentable al tiempo que satisface mayores estándares de calidad y compromisos ambientales internacionales. Asimismo, ha reformado su marco institucional para enfrentar de forma más eficiente los retos del sector en sus dos vertientes: la pesca marítima comercial y la producción acuícola de especies de alto valor. No obstante que las políticas gubernamentales han mejorado el nivel de rentabilidad del sector $\mathrm{y}$ han propiciado un cierto nivel del desarrollo, el apoyo gubernamental no ha cubierto todas las necesidades de apoyo. El crecimiento de la industria requiere apoyos financieros, pero también de manera importante, normatividad que la fomente, y planeación y legislación adecuada. 


\section{Conclusión}

El balance derivado del análisis de la evidencia empírica presentada a lo largo del presente trabajo muestra que los gobiernos y organizaciones en todos los niveles reconocen la importancia del sector pesquero y su potencial para fomentar el desarrollo local. Así mismo, el análisis detambién mostró que movilizar el sector con el objetivo de aprovechar los recursos pesqueros puede impactar positivamente sobre el bienestar de la población que se encuentra inmersa en la dinámica económica de los espacios locales costeros y rurales de México y del estado de Nayarit, como en el caso particular del municipio de San Blas que fue el objeto de estudio en este trabajo de investigación. San Blas es un municipio que posee gran abundancia de recursos naturales con potencial de desarrollo que se traduciría en beneficios económicos y sociales para la población.

La evidencia muestra que dada la importancia socioeconómica del sector, el impacto de las políticas públicas sobre la actividad es todavía tangencial y subestima la oportunidad que representa para el desarrollo San Blas, Nayarit. A pesar de que San Blas se encuentra entre los tres municipios destacados en el sector pesquero a nivel municipal, ha tenido un lento desarrollo debido a la falta de infraestructura y procesamiento del producto, métodos obsoletos para la captura y escasez de vías de acceso; otras causas son falta de insumos, falta de diversificación ya que la captura está concentrada en el camarón y el ostión; asimismo la falta de organización de los productores y la inadecuada comercialización de los productos, irregularidades en la venta de la producción, ocasionando altos precios al consumidor.Lo anterior saca a la luz que a pesar de existir políticas específicas para el sector, los resultados no son los planteados en los planes y programas que sirven de instrumento para la aplicación de políticas públicas, de esta forma, podría decirse que existe un fallo en la implementación y ejecución de las políticas y una falta de control y evaluación. Dejando rezagado a un sector con alto potencial de desarrollo. 


\section{Bibliografía}

Pesquera Álvarez, Torres Porfirio, C. Ramírez Martínez, A. Orbe Mendoza (1999). Desarrollo de la acuacultura en México y perspectivas de la Acuacultura rural. Taller ARPE, FAO-UCT.

Poggie, J.J., y Pollnac, R.B. (Editors). 1991. Small-scale fishery development: sociocultural aspects. International Center for Marine Resource Development (ICMRD). TheUniversity of Rhode Island. Kingston, RI. USA.

Rodríguez-Valencia, J.A. \& M.A. Cisneros-Mata. 2006. Captura incidental de las flotas Pesqueras ribereñas del Pacífico Mexicano. [Reporte técnico] Programa Golfo de California de WWF-México a la Comisión de Cooperación Ambiental de América del Norte. Recuperado dehttp://www.wwf.org.mx/wwfmex/publicaciones.php?tipo=reps

Unzueta Bustamante Marco Linné (2009). La Acuicultura frente al reto de la globalización alimenticia. Ciencia Pesquera. 17 (2). 